01

\title{
Исследование соотношений взаимности для нелинейного многополюсника в неоднородном магнитном поле
}

\author{
(C) В.К. Игнатьев, С.В. Перченко \\ Волгоградский государственный университет, \\ 400062 Волгоград, Россия \\ ฯ e-mail: perchenko.sergey@gmail.com
}

(Поступило в Редакцию 20 июня 2016 г. В окончательной редакции 9 ноября 2016 г.)

На основе материального уравнения нелинейной неоднородной и нестационарной проводящей среды в приближении интеграла столкновений Ландау получены соотношения взаимности для матрицы нелинейных сопротивлений многополюсника, находящегося в неоднородном внешнем магнитном поле. Рассмотрен вопрос об измеряемых потенциалах выводов многополюсника в квазистационарном режиме. Предложен способ проверки полученных соотношений взаимности и приведены экспериментальные результаты. Показано, что в пределах погрешности электрических измерений соотношения взаимности для нелинейного многополюсника выполняются.

DOI: 10.21883/JTF.2017.06.44504.1942

\section{Введение}

Элементы магнетоэлектроники в общем случае можно рассматривать как параметрические многополюсники, осуществляющие интегральное преобразование сигналов вида свертки, ядром которого является системная функция, полностью описывающая все свойства элемента [1]. Сигнальным воздействием на такой многополюсник является в общем случае неоднородное внешнее магнитное поле, которое может быть существенно меньшим, чем магнитные поля, создаваемые протекающими по многополюснику токами. Выделить реакцию многополюсника на малое внешнее поле можно методом взаимности [2]. Однако преобразователи, применяемые в устройствах функциональной электроники, имеют нелинейные вольт-амперные характеристики. Соотношения же Онзагера симметрии кинетических коэффициентов [3] получены в линейном приближении для однородного магнитного поля. Возможность их применения для нелинейных систем требует обоснования.

Для стационарной, однородной и нелинейной проводящей среды, находящейся в однородном магнитном поле, в релаксационном приближении были получены соотношения взаимности для тензора нелинейной проводимости [4]. Однако их экспериментальная проверка не представляется возможной, так как измерительные зонды, вводимые в проводящую среду, исказят однородность полей в ней. Кроме того, протекающие токи создают в среде неоднородное магнитное поле. Без теоретического обоснования предложены соотношения взаимности для матрицы электрически измеряемых нелинейных сопротивлений четырехполюсника и проведена их экспериментальная проверка [5].

Получить универсальные соотношения взаимности для нелинейной системы в неоднородном магнитном поле без предположений о процессах в ней, видимо, не возможно [6]. Даже для неравновесных процессов в ли- нейных системах соотношения взаимности постулируются [7]. Поэтому представляет интерес обосновать такие соотношения в наиболее общих предположениях для измеряемых с наивысшей точностью без возмущения процессов в самой системе величин. Такой системой может быть нелинейный многополюсник, процессы переноса в котором описываются кинетическим уравнением.

\section{Материальное уравнение нелинейной среды}

Основным механизмом релаксации носителей заряда проводимости является рассеяние на ионах, коллектив которых является термостатом для носителей заряда. Функция распределения $f_{r}\left(t, \mathbf{r}, \mathbf{p}^{\prime}, R\right)$ ионов равновесная, определяется только температурой и не зависит от электрического и магнитного полей, а скорость ионов много меньше скорости носителей заряда проводимости. Тогда процессы переноса в такой среде описываются кинетическим уравнением Власова [8]

$$
\frac{\partial f_{\alpha}}{\partial t}+\mathbf{v}_{\alpha} \frac{\partial f_{\alpha}}{\partial \mathbf{r}}+q_{\alpha}\left(\mathbf{E}+\mathbf{v}_{\alpha} \times \mathbf{B}\right) \frac{\partial f_{\alpha}}{\partial \mathbf{p}}=\hat{I}_{\alpha}\left(f_{\alpha}, f_{r}\right),
$$

где

$$
\begin{aligned}
& \hat{I}_{\alpha}=-\pi q_{\alpha}^{2} q_{r}^{2} L \frac{\partial}{\partial p_{i}} \int\left[f_{\alpha}(t, \mathbf{r}, \mathbf{p}) \frac{\partial f_{r}\left(t, \mathbf{r}, \mathbf{p}^{\prime}\right)}{\partial p_{j}^{\prime}}\right. \\
& \left.-f_{r}\left(t, \mathbf{r}, \mathbf{p}^{\prime}\right) \frac{\partial f_{\alpha}(t, \mathbf{r}, \mathbf{p})}{\partial p_{j}}\right]\left(\delta_{i j}-\frac{p_{i}^{\prime} p_{j}^{\prime}}{p^{\prime 2}}\right) \frac{m_{\alpha} d^{3} p^{\prime}}{p^{\prime}}
\end{aligned}
$$

- интеграл столкновений Ландау, $q_{r}$ - заряд ионов решетки, $f_{\alpha}(t, \mathbf{r}, \mathbf{p})$ - функция распределения носителей заряда $q_{\alpha}, \mathbf{v}_{\alpha}=\mathbf{p} / m_{\alpha}-$ их скорость, $m_{\alpha}-$ их эффективная масса, $L-$ кулоновский логарифм. В дальнейшем зависимость функций распределения от температуры предполагается, но явно не указывается. Поскольку ионы 
решетки не участвуют в процессах переноса, их функция распределения $f_{r}\left(t, \mathbf{r}, \mathbf{p}^{\prime}\right)$ рассматривается как независимая. Поэтому интеграл столкновений (2) линейно зависит от $f_{\alpha}(t, \mathbf{r}, \mathbf{p})$, и интегрально-дифференциальное уравнение (1) является линейным относительно функции распределения носителей заряда $f_{\alpha}(t, \mathbf{r}, \mathbf{p})$.

В устойчивом состоянии среды, не испытывающей внешних воздействий, кроме электрического и магнитного полей и тех, которые могут быть описаны функцией распределения решетки $f_{r}\left(t, \mathbf{r}, \mathbf{p}^{\prime}\right)$, функции распределения $f_{\alpha}(t, \mathbf{r}, \mathbf{p})$ для каждого сорта носителей заряда однозначно определяются заданными магнитным и электрическими полями. Это означает единственность решения интегрально-дифференциального уравнения (1).

Рассмотрим случай, когда поля Е и В постоянные, однородные, не нулевые и не сонаправленные, и введем новые переменные

$$
\begin{gathered}
y_{1}=\mathbf{r E}, \quad y_{2}=\mathbf{r B}, \quad y_{3}=\mathbf{r}[\mathbf{E} \times \mathbf{B}], \\
z_{1}=\mathbf{p E}, \quad z_{2}=\mathbf{p B}, \quad z_{3}=\mathbf{p}[\mathbf{E} \times \mathbf{B}], \\
z_{1}^{\prime}=\mathbf{p}^{\prime} \mathbf{E}, \quad z_{2}^{\prime}=\mathbf{p}^{\prime} \mathbf{B}, \quad z_{3}^{\prime}=\mathbf{p}^{\prime}[\mathbf{E} \times \mathbf{B}] .
\end{gathered}
$$

Выразим импульс р и координату $\mathbf{r}$ как функцию векторов Е и В, а также переменных (3) и (4), которые являются скалярами и не преобразуются при поворотах системы координат:

$$
\begin{aligned}
\mathbf{r} & =\frac{B^{2} y_{1}-\mathbf{E B} y_{2}}{|\mathbf{E} \times \mathbf{B}|^{2}} \mathbf{E}+\frac{E^{2} y_{2}-\mathbf{E B} y_{1}}{|\mathbf{E} \times \mathbf{B}|^{2}} \mathbf{B}+\frac{y_{3}[\mathbf{E} \times \mathbf{B}]}{|\mathbf{E} \times \mathbf{B}|^{2}}, \\
\mathbf{p} & =\frac{B^{2} z_{1}-\mathbf{E B} z_{2}}{|\mathbf{E} \times \mathbf{B}|^{2}} \mathbf{E}+\frac{E^{2} z_{2}-\mathbf{E B} z_{1}}{|\mathbf{E} \times \mathbf{B}|^{2}} \mathbf{B}+\frac{z_{3}}{|\mathbf{E} \times \mathbf{B}|^{2}}[\mathbf{E} \times \mathbf{B}], \\
\mathbf{p}^{\prime} & =\frac{B^{2} z_{1}^{\prime}-\mathbf{E B} z_{2}^{\prime}}{|\mathbf{E} \times \mathbf{B}|^{2}} \mathbf{E}+\frac{E^{2} z_{2}^{\prime}-\mathbf{E B} z_{1}^{\prime}}{|\mathbf{E} \times \mathbf{B}|^{2}} \mathbf{B}+\frac{z_{3}^{\prime}}{|\mathbf{E} \times \mathbf{B}|^{2}}[\mathbf{E} \times \mathbf{B}] .
\end{aligned}
$$

Преобразования (3)-(6) взаимно однозначны и

$$
\begin{gathered}
\frac{\partial f_{\alpha}}{\partial \mathbf{r}}=\mathbf{E} \frac{\partial F_{\alpha}}{\partial y_{1}}+\mathbf{B} \frac{\partial F_{\alpha}}{\partial y_{2}}+[\mathbf{E} \times \mathbf{B}] \frac{\partial F_{\alpha}}{\partial y_{3}}, \\
\frac{\partial f_{\alpha}}{\partial \mathbf{p}}=\mathbf{E} \frac{\partial F_{\alpha}}{\partial z_{1}}+\mathbf{B} \frac{\partial F_{\alpha}}{\partial z_{2}}+[\mathbf{E} \times \mathbf{B}] \frac{\partial F_{\alpha}}{\partial z_{3}}, \\
\frac{\partial f_{r}}{\partial \mathbf{p}^{\prime}}=\mathbf{E} \frac{\partial F_{r}}{\partial z_{1}^{\prime}}+\mathbf{B} \frac{\partial F_{r}}{\partial z_{2}^{\prime}}+[\mathbf{E} \times \mathbf{B}] \frac{\partial F_{r}}{\partial z_{3}^{\prime}},
\end{gathered}
$$

где функции распределения $F_{\alpha}(t, \mathbf{y}, \mathbf{z})$ получаются из функций $f_{\alpha}(t, \mathbf{r}, \mathbf{p})$ заменой переменных (5) и (6). При этом

$$
\begin{aligned}
& f_{\alpha}(t, \mathbf{r}, \mathbf{p})=F_{\alpha}\left(t, y_{1}, y_{2}, y_{3}, z_{1}, z_{2}, z_{3}\right), \\
& f_{r}\left(t, \mathbf{r}, \mathbf{p}^{\prime}\right)=F_{r}\left(t, y_{1}, y_{2}, y_{3}, z_{1}^{\prime}, z_{2}^{\prime}, z_{3}^{\prime}\right) .
\end{aligned}
$$

Тогда уравнение (1) с учетом формул (3), (7)-(9) преобразуется к виду

$$
\begin{aligned}
\frac{\partial F_{\alpha}}{\partial t} & +\frac{z_{1}}{m_{\alpha}} \frac{\partial F_{\alpha}}{\partial y_{1}}+\frac{z_{2}}{m_{\alpha}} \frac{\partial F_{\alpha}}{\partial y_{2}}+\frac{z_{3}}{m_{\alpha}} \frac{\partial F_{\alpha}}{\partial y_{3}} \\
& +q_{\alpha} E^{2} \frac{\partial F_{\alpha}}{\partial z_{1}}+\left(E^{2}+\frac{y_{3}}{m_{\alpha}}\right) \frac{\partial F_{\alpha}}{\partial z_{1}}+\mathbf{E B} \frac{\partial F_{\alpha}}{\partial z_{2}} \\
& +\left(B^{2} y_{1}-\mathbf{E B} y_{2}\right) \frac{\partial F_{\alpha}}{\partial z_{3}}=\tilde{I}_{\alpha}\left(F_{\alpha}, F_{r}\right) .
\end{aligned}
$$

Здесь оператор $\tilde{I}_{\alpha}\left(F_{\alpha}, F_{r}\right)$ получается из оператора (2) заменой переменных (5) и (6). С учетом формул (4) якобиан преобразований (6) $J=|\mathbf{E} \times \mathbf{B}|^{-2}$ и модуль импульса инвариантны относительно одновременной инверсии полей $\mathbf{E}$ и $\mathbf{B}$ :

$$
\begin{gathered}
J(-\mathbf{E},-\mathbf{B})=J(\mathbf{E}, \mathbf{B}), \\
p^{\prime}\left(z_{1}^{\prime}, z_{2}^{\prime}, z_{3}^{\prime},-\mathbf{E},-\mathbf{B}\right)=p^{\prime}\left(z_{1}^{\prime}, z_{2}^{\prime}, z_{3}^{\prime}, \mathbf{E}, \mathbf{B}\right) .
\end{gathered}
$$

Преобразуем производные функций распределения в интеграле столкновений (2) с учетом формул (4) и (8)

$$
\begin{aligned}
& p_{i}^{\prime} p_{j}^{\prime} \frac{\partial^{2} f_{\alpha}}{\partial p_{i} \partial p_{j}}=z_{1}^{\prime 2} \frac{\partial^{2} F_{\alpha}}{\partial z_{1}^{2}}+z_{2}^{\prime 2} \frac{\partial^{2} F_{\alpha}}{\partial z_{2}^{2}}+z_{3}^{\prime 2} \frac{\partial^{2} F_{\alpha}}{\partial z_{3}^{2}} \\
& \quad+2 z_{1}^{\prime} z_{2}^{\prime} \frac{\partial^{2} F_{\alpha}}{\partial z_{1} \partial z_{2}}+2 z_{1}^{\prime} z_{3}^{\prime} \frac{\partial^{2} F_{\alpha}}{\partial z_{1} \partial z_{3}}+2 z_{2}^{\prime} z_{3}^{\prime} \frac{\partial^{2} F_{\alpha}}{\partial z_{2} \partial z_{3}} .
\end{aligned}
$$

Остальные слагаемые в интеграле столкновений (2) вычисляются аналогично. Тогда из формулы (11) следует, что интеграл столкновений в правой части уравнения (10) тоже инвариантен относительно одновременной инверсии полей $\mathbf{E}$ и $\mathbf{B}$ :

$$
\tilde{I}_{\alpha}(t, \mathbf{r},-\mathbf{E},-\mathbf{B})=\tilde{I_{\alpha}}(t, \mathbf{r}, \mathbf{E}, \mathbf{B}) .
$$

Следовательно, уравнение (10) в новых переменных (3) и (4) инвариантно относительно одновременной инверсии полей $\mathbf{E}$ и $\mathbf{B}$.

Ограничимся рассмотрением устойчивых состояний среды. Тогда единственное решение инвариантного относительно одновременной инверсии полей $\mathbf{E}$ и $\mathbf{B}$ уравнения (10) также инвариантно относительно одновременной инверсии полей $\mathbf{E}$ и $\mathbf{B}$ :

$$
F_{\alpha}(t, \mathbf{y}, \mathbf{z},-\mathbf{E},-\mathbf{B})=F_{\alpha}(t, \mathbf{y}, \mathbf{z}, \mathbf{E}, \mathbf{B}) .
$$

Выполним в материальном уравнении среды

$$
\mathbf{j}(t, \mathbf{r})=\sum_{\alpha} \int \frac{q_{\alpha} c_{\alpha} \mathbf{p}}{m_{\alpha}} f_{\alpha}(t, \mathbf{r}, \mathbf{p}) d^{3} p,
$$

где $c_{\alpha}-$ концентрация носителей заряда $q_{\alpha}$, замену переменных (6) с учетом формулы (9)

$$
\begin{aligned}
\mathbf{j}(t, \mathbf{r})=K_{1}(t, \mathbf{r}, \mathbf{E}, \mathbf{B}) \mathbf{E} & +K_{2}(t, \mathbf{r}, \mathbf{E}, \mathbf{B}) \mathbf{B} \\
& +K_{3}(t, \mathbf{r}, \mathbf{E}, \mathbf{B})[\mathbf{E} \times \mathbf{B}],
\end{aligned}
$$


где

$$
\begin{aligned}
K_{1}(t, \mathbf{r}, \mathbf{E}, \mathbf{B})= & \frac{1}{|\mathbf{E} \times \mathbf{B}|^{4}} \sum_{\alpha} \frac{c_{\alpha} q_{\alpha}}{m_{\alpha}} \\
& \times \int\left\{B^{2} z_{1}-\mathbf{E B} z_{2}\right\} F_{\alpha}(t, \mathbf{y}, \mathbf{z}) d^{3} z, \\
K_{2}(t, \mathbf{r}, \mathbf{E}, \mathbf{B})= & \frac{1}{|\mathbf{E} \times \mathbf{B}|^{4}} \sum_{\alpha} \frac{c_{\alpha} q_{\alpha}}{m_{\alpha}} \\
& \times \int\left\{E^{2} z_{2}-\mathbf{E B} y_{1}\right\} F_{\alpha}(t, \mathbf{y}, \mathbf{z}) d^{3} z, \\
K_{3}(t, \mathbf{r}, \mathbf{E}, \mathbf{B})= & \frac{1}{|\mathbf{E} \times \mathbf{B}|^{4}} \sum_{\alpha} \frac{c_{\alpha} q_{\alpha}}{m_{\alpha}} \int z_{3} F_{\alpha}(t, \mathbf{y}, \mathbf{z}) d^{3} z .
\end{aligned}
$$

Из формул (12) и (13) следует, что

$$
K_{n}(t, \mathbf{r}, \mathbf{E}, \mathbf{B})=K_{n}(t, \mathbf{r},-\mathbf{E},-\mathbf{B}), \quad n=1,2,3 .
$$

Коэффициенты $K_{n}(\mathbf{E}, \mathbf{B})$ не определены при $\mathbf{E}=0$ и $\mathbf{B}=0$. В слабом электрическом поле $|\mathbf{E}| \ll E_{T}=\varphi_{T} / l_{T}$, где $\varphi_{T}=k T / q-$ тепловой потенциал основных носителей заряда, материальное уравнение стационарной, однородной и изотропной среды в магнитном поле имеет вид закона Ома, в котором к силе Кулона добавлена сила Лоренца

$$
\mathbf{j}=c q v\left(\mathbf{E}+\frac{1}{c q}[\mathbf{j} \times \mathbf{B}]\right),
$$

где $v$ - подвижность основных носителей заряда. Решая это уравнение относительно вектора плотности тока $\mathbf{j}$, получим

$$
\mathbf{j}=\frac{\sigma}{1+|v \mathbf{B}|^{2}} \mathbf{E}+\frac{\sigma v^{2}(\mathbf{E B})}{1+|v \mathbf{B}|^{2}} \mathbf{B}+\frac{\sigma v}{1+|v \mathbf{B}|^{2}}[\mathbf{E} \times \mathbf{B}],
$$

где $\sigma=c q v-$ проводимость среды в нулевых электрическом и магнитном полях.

Сравнивая соотношения (13) и (14), запишем материальное уравнение нелинейной среды в виде

$$
\begin{aligned}
\mathbf{j}(t, \mathbf{r})= & \sigma\left\{1+\alpha\left(t, \mathbf{r}, \mathbf{E} / E_{T}, v \mathbf{B}\right)\right\} \mathbf{E} \\
& +\sigma v E_{T} \beta\left(t, \mathbf{r}, \mathbf{E} / E_{T}, v \mathbf{B}\right) \mathbf{B} \\
& +\sigma v \gamma\left(t, \mathbf{r}, \mathbf{E} / E_{T}, v \mathbf{B}\right)[\mathbf{E} \times \mathbf{B}] .
\end{aligned}
$$

Здесь

$$
\begin{gathered}
\alpha(t, \mathbf{r}, \mathbf{E}=0, \mathbf{B}=0)=\beta(t, \mathbf{r}, \mathbf{E}=0, \mathbf{B}=0)=0, \\
\gamma(t, \mathbf{r}, \mathbf{E}=0, \mathbf{B}=0)=1,
\end{gathered}
$$

и в соответствии с формулой (11)

$$
\begin{aligned}
& \alpha(t, \mathbf{r},-\mathbf{E},-\mathbf{B})=\alpha(t, \mathbf{r}, \mathbf{E}, \mathbf{B}), \\
& \beta(t, \mathbf{r},-\mathbf{E},-\mathbf{B})=\beta(t, \mathbf{r}, \mathbf{E}, \mathbf{B}), \\
& \gamma(t, \mathbf{r},-\mathbf{E},-\mathbf{B})=\gamma(t, \mathbf{r}, \mathbf{E}, \mathbf{B}) .
\end{aligned}
$$

\section{Квазистационарное приближение}

Рассмотрим обобщенную конструкцию объемного многополюсника $D$ с линейными размерами, много большими $l_{T}$, где $l_{T}-$ средняя длина свободного пробега носителей заряда, с $M$ контактами $\left(S_{m}, m=1,2, \ldots, M\right)$. Пусть $\mathbf{B}=\mathbf{B}_{e}+\mathbf{B}^{\prime}-$ полная индукция магнитного поля в элементе, $\mathbf{B}_{e}-$ поле, созданное внешними источниками, $\mathbf{B}^{\prime}$ - поле токов, текущих через многополюсник. При этом

$$
\operatorname{rot} \mathbf{B}_{e}=0, \quad \operatorname{rot} \mathbf{B}^{\prime}=\mu_{0} \mathbf{j} .
$$

Материальное уравнение (15) получено для однородных и постоянных электрического и магнитного полей и является локальным. Нелокальность проявляется, когда поля существенно меняются на расстоянии порядка длины сводного пробега. Если $\left|\partial E_{i} / \partial r_{j}\right| \ll|\mathbf{E}| / l_{T}$, $\left|\partial B_{i} / \partial r_{j}\right| \ll|\mathbf{B}| / l_{T}, i, j=1,2,3$, эффектом нелокальности можно пренебречь и считать, что

$|\operatorname{grad} \alpha| \ll|\alpha| / l_{T},|\operatorname{grad} \beta| \ll|\beta| / l_{T},|\operatorname{grad} \gamma| \ll|\gamma| / l_{T}$.

В квазистационарном случае, когда ток смещения много меньше тока проводимости, диэлектрическая и магнитная проницаемости среды являются константами, уравнение непрерывности $\operatorname{div} \mathbf{j}=0$ с учетом материального уравнения (15) в безразмерных координатах $\mathbf{x}=\mathbf{r} / l_{T}$ принимает вид

$$
\Delta \varphi=-\mu_{0} \sigma v \gamma|\nabla \varphi|^{2}-\frac{\mu_{0} \varphi_{T} \sigma v \beta \gamma}{1+\alpha} \nabla(\varphi) \mathbf{b},
$$

где

$$
\mathbf{E}=-\operatorname{grad} \varphi-\frac{\partial \mathbf{A}}{\partial t}, \quad \mathbf{B}=\operatorname{rot} \mathbf{A},
$$

$\varphi$ - электростатический потенциал, $\mathbf{b}=v \mathbf{B}, \mathbf{A}-$ векторный потенциал в кулоновской калибровке $\operatorname{div} \mathbf{A}=0$, а оператор $\nabla$ берется по безразмерным координатам $\mathbf{x}$. Второе уравнение (17) в кулоновской калибровке принимает вид $\Delta \mathbf{A}=-\mu_{0} \mathbf{j}$, его решение

$$
\mathbf{A}(\mathbf{r})=\frac{\mu_{0}}{4 \pi} \int_{D} \frac{\mathbf{j}\left(\mathbf{r}^{\prime}\right)}{\left|\mathbf{r}-\mathbf{r}^{\prime}\right|} d^{3} r
$$

Пусть в область $D$, ограниченную поверхностью $S$, через соответствующие контактные поверхности $S_{m}$ втекают токи $I_{m}$. Условие для нормальной компоненты плотности тока на границе $S$ имеет вид

$$
\left.j_{n}(\mathbf{x})\right|_{S}=-\frac{1}{l_{T}^{2}} \sum_{m=1}^{M} I_{m} g_{m}(\mathbf{x}),
$$

где $g_{m}(\mathbf{x})-$ функция-носитель поверхности $S_{m}$ :

$$
\oint_{S_{m}} g_{m}(\mathbf{x}) d s=1, \quad g_{m}\left(\mathbf{x} \notin S_{m}\right) \equiv 0 .
$$

Из уравнения (18) в этом случае следует, что распределение потенциала является решением внутренней 
смешанной задачи, когда на границе области задана линейная комбинация нормальной и тангенциальной производных потенциала

$$
\begin{aligned}
& \left((1+\alpha) \frac{\partial \varphi}{\partial n}-\beta v \varphi_{T} \mathbf{B n}+\gamma v\left[\frac{\partial \varphi}{\partial \mathbf{x}} \times \mathbf{B}\right] \mathbf{n}\right. \\
& \left.+(1+\alpha) \frac{\partial \mathbf{A}}{\partial t} \mathbf{n}\right)\left.\right|_{S}=-\frac{1}{\sigma l_{T}} \sum_{m=1}^{M} I_{m} g_{m}(\mathbf{x}),
\end{aligned}
$$

где $\mathbf{n}-$ внешняя нормаль поверхности $S$.

Примем за ноль средний по поверхности $S$ тела $D$ электростатический потенциал и будем искать совместное решение уравнений (18) и (20) с граничными условиями (23) методом последовательных приближений. Перейдем к безразмерным величинам

$$
\begin{gathered}
\varphi(\mathbf{x})=\varphi_{T} \psi(\mathbf{x}), \quad \psi(\mathbf{x})=\psi_{0}(\mathbf{x})+\delta \psi_{1}(\mathbf{x})+\ldots, \\
\mathbf{E}(\mathbf{x})=\varphi_{T} \mathbf{e}(\mathbf{x}) / l_{T}, \quad \mathbf{A}(\mathbf{x})=l_{T} \mathbf{a}(\mathbf{x}) / v \\
\mathbf{e}(\mathbf{x})=\mathbf{e}_{0}(\mathbf{x})+\delta \mathbf{e}_{1}(\mathbf{x})+\ldots \\
\mathbf{a}(\mathbf{x})=\mathbf{a}_{0}(\mathbf{x})+\delta \mathbf{a}_{1}(\mathbf{x})+\ldots \\
\mathbf{b}^{\prime}(\mathbf{x})=\mathbf{b}_{0}(\mathbf{x})+\delta \mathbf{b}_{1}(\mathbf{x})+\ldots
\end{gathered}
$$

где

$$
\begin{gathered}
\delta=\mu_{0} \varphi_{T} \sigma v, \quad 0<\delta<1, \quad \alpha_{0}=\alpha\left(\mathbf{e}_{0}, \mathbf{b}_{0}\right), \\
\beta_{0}=\beta\left(\mathbf{e}_{0}, \mathbf{b}_{0}\right), \quad \gamma_{0}=\gamma\left(\mathbf{e}_{0}, \mathbf{b}_{0}\right) .
\end{gathered}
$$

В линейном по слабому внешнему полю $\left|\mathbf{B}_{e}\right| \ll 1 / v$ приближении уравнения (18) и (23) эквивалентны цепочке краевых задач Неймана для уравнений Лапласа и Пуассона,

$$
\begin{gathered}
\Delta \psi_{0}=0,\left.\quad \frac{\partial \psi_{0}}{\partial n}\right|_{S}=-\frac{1}{I_{T}} \sum_{m=1}^{M} I_{m} g_{m}(\mathbf{x}), \\
\nabla \times \mathbf{b}_{0}=-\delta\left(1+\alpha_{0}\right) \nabla \psi_{0}+\delta \beta_{0} \mathbf{b}_{0}-\delta \gamma_{0}\left[\nabla \psi_{0} \times \mathbf{b}_{0}\right], \\
\nabla \mathbf{b}_{0}=0, \quad \nabla \times \mathbf{b}_{e}=0, \quad \nabla \mathbf{b}_{e}=0, \\
\nabla \psi_{1}=-\gamma_{0}\left|\nabla \psi_{0}\right|^{2}-\frac{\gamma_{0} \beta_{0}}{1+\alpha_{0}}\left(\mathbf{b}_{0}+\mathbf{b}_{e}\right) \nabla \psi_{0}, \\
\left.\frac{\partial \psi_{1}}{\partial n}\right|_{S}=-\left.\frac{\gamma_{0}}{\delta\left(1+\alpha_{0}\right)}\left[\frac{\partial \psi_{0}}{\partial \mathbf{x}} \times\left(\mathbf{b}_{0}+\mathbf{b}_{e}\right)\right] n\right|_{S} \\
+\left.\frac{\beta_{0}\left(\mathbf{b}_{0}+\mathbf{b}_{e}\right) \mathbf{n}}{\delta\left(1+\alpha_{0}\right)}\right|_{S}-\left.\frac{\alpha_{0} \mathbf{n}}{\delta\left(1+\alpha_{0}\right)} \frac{\partial \psi_{0}}{\partial \mathbf{x}}\right|_{S}-\left.\frac{\partial \mathbf{a n}}{\partial \tau}\right|_{S},
\end{gathered}
$$

где обозначено $I_{T}=\sigma \varphi_{T} l_{T}, \tau=\frac{v \varphi_{T}}{l_{T}^{2}} t-$ безразмерное время.

Решение внутренней краевой задачи (24) при выполнении вытекающего из первого закона Кирхгофа условия

$$
\oint_{S} \sum_{m=1}^{M} I_{m} g_{m}(\mathbf{x}) d s=0
$$

существует и имеет вид

$$
\psi_{0}(\mathbf{x})=\frac{1}{I_{T}} \sum_{m=1}^{M} I_{m} \oint_{S} G\left(\mathbf{x}, \mathbf{x}^{\prime}\right) g_{m}\left(\mathbf{x}^{\prime}\right) d s^{\prime}+C,
$$

где $G\left(\mathbf{x} \in D, \mathbf{x}^{\prime} \in D\right)$ - функция Грина задачи Неймана для области $\mathrm{D}$, ограниченной поверхностью $S, C$ константа, которая определяется при выборе нулевого потенциала [9]. Поскольку за ноль принят средний по поврехности тела $S$ потенциал, из формул (21) и (27) следует, что

$$
-\frac{l_{T}^{2}}{I_{T}} \oint_{S}\left[\oint_{S} G\left(\mathbf{x}, \mathbf{x}^{\prime}\right) d s\right] j_{n}\left(\mathbf{x}^{\prime}\right) d s^{\prime}+C S=0 .
$$

При выполнении дополнительного условия

$$
\oint_{S} G\left(\mathbf{x}, \mathbf{x}^{\prime}\right) d s=0
$$

получаем $C=0$, а функция Грина задачи Неймана, удовлетворяющая этому условию, является единственной и симметричной [9]:

$$
G\left(\mathbf{x}, \mathbf{x}^{\prime}\right)=G\left(\mathbf{x}^{\prime}, \mathbf{x}\right)
$$

Поскольку поле $\mathbf{B}^{\prime}(\mathbf{r})$ создается только токами в области $D$, уравнение (20) для безразмерных величин в первом приближении, когда холловский ток много меньше тока проводимости, имеет вид

$$
\begin{gathered}
\mathbf{a}_{0}(\mathbf{x})=-\frac{\delta}{4 \pi} \int_{D} \frac{\partial \psi_{0}\left(\mathbf{x}^{\prime}\right) / \partial \mathbf{x}^{\prime}}{\left|\mathbf{x}-\mathbf{x}^{\prime}\right|} d^{3} x^{\prime}, \\
\mathbf{b}_{0}(\mathbf{x})=-\frac{\delta}{4 \pi} \int_{D}\left[\frac{\partial \psi_{0}\left(\mathbf{x}^{\prime}\right)}{\partial \mathbf{x}^{\prime}} \times \frac{\mathbf{x}-\mathbf{x}^{\prime}}{\left|\mathbf{x}-\mathbf{x}^{\prime}\right|^{3}}\right] d^{3} x^{\prime} .
\end{gathered}
$$

Введем $M$-мерный вектор $\mathbf{I}$ с компонентами $I_{1}, \ldots, I_{M}$. Из уравнений (27) следует, что $\psi_{0}(\mathbf{x}, \mathbf{I})=$ $=-\psi_{0}(\mathbf{x},-\mathbf{I})$. Тогда с учетом формул (19) и (28) получим

$$
\begin{gathered}
\mathbf{a}_{0}(\mathbf{x}, \mathbf{I})=\mathbf{a}_{0}(\mathbf{x},-\mathbf{I}), \quad \mathbf{b}_{0}(\mathbf{x}, \mathbf{I})=\mathbf{b}_{0}(\mathbf{x},-\mathbf{I}), \\
\mathbf{e}_{0}(\mathbf{x}, \mathbf{I})=\mathbf{e}_{0}(\mathbf{x},-\mathbf{I}), \\
\alpha_{0}(\mathbf{x}, \mathbf{I})=\alpha_{0}(\mathbf{x},-\mathbf{I}), \quad \beta_{0}(\mathbf{x}, \mathbf{I})=\beta_{0}(\mathbf{x},-\mathbf{I}), \\
\gamma_{0}(\mathbf{x}, \mathbf{I})=\gamma_{0}(\mathbf{x},-\mathbf{I}), \\
\mathbf{e}_{0}(\mathbf{x}, 0)=0, \quad \mathbf{b}_{0}(\mathbf{x}, 0)=0, \\
\alpha_{0}(\mathbf{x}, 0)=0, \quad \beta_{0}(\mathbf{x}, 0)=0, \quad \gamma_{0}(\mathbf{x}, 0)=1 .
\end{gathered}
$$

Если многополюсник $D$ электрически нейтрален, то поток вектора е 0 через его поверхность $S$ по теореме 
Гаусса равен нулю. Поток вектора $\mathbf{b}_{0}$ через поверхность $S$ тела всегда равен нулю. Тогда с учетом уравнений (15), (19) и (25) в кулоновской калибровке получаем

$$
\begin{aligned}
& \frac{1}{\delta} \oint_{S} \frac{\gamma_{0}}{1+\alpha_{0}}\left[\nabla \psi_{0} \times\left(\mathbf{b}_{0}+\mathbf{b}_{e}\right)\right] d \mathbf{s}+\oint_{S} \frac{\partial \mathbf{a}}{\partial \tau} d \mathbf{s} \\
& =\int_{D} \frac{\gamma_{0}}{1+\alpha_{0}}\left|\nabla \psi_{0}\right|^{2} d^{3} r+\int_{D} \frac{\gamma_{0} \beta_{0}}{1+\alpha_{0}}\left(\nabla \psi_{0}\right)\left(\mathbf{b}_{0}+\mathbf{b}_{e}\right) d^{3} r .
\end{aligned}
$$

Таким образом, условие разрешимости краевой задачи (26) выполняется, и ее решение имеет вид [9]

$$
\begin{aligned}
& \psi_{1}(\mathbf{x})=\int_{D} \gamma_{0}\left(\mathbf{x}^{\prime}\right) G\left(\mathbf{x}, \mathbf{x}^{\prime}\right)\left\{\left|\frac{\partial \psi_{0}}{\partial \mathbf{x}^{\prime}}\right|^{2}+\frac{1}{\delta} \frac{\beta_{0}\left(\mathbf{x}^{\prime}\right)}{1+\alpha_{0}\left(\mathbf{x}^{\prime}\right)}\right. \\
& \left.\times \frac{\partial \psi_{0}}{\partial \mathbf{x}^{\prime}}\left(\mathbf{b}_{0}\left(\mathbf{x}^{\prime}\right)+\mathbf{b}_{e}\left(\mathbf{x}^{\prime}\right)\right)\right\} d^{3} x^{\prime}+\frac{1}{\delta} \oint_{S} \frac{\alpha_{0}\left(\mathbf{x}^{\prime}\right)}{1+\alpha_{0}\left(\mathbf{x}^{\prime}\right)} G\left(\mathbf{x}, \mathbf{x}^{\prime}\right) \\
& \times \frac{\partial \psi_{0}}{\partial \mathbf{x}^{\prime}} d \mathbf{s}^{\prime}-\frac{1}{\delta} \oint_{S} \frac{\beta_{0}\left(\mathbf{x}^{\prime}\right)}{1+\alpha_{0}\left(\mathbf{x}^{\prime}\right)} G\left(\mathbf{x}, \mathbf{x}^{\prime}\right)\left(\mathbf{b}_{0}\left(\mathbf{x}^{\prime}\right)+\mathbf{b}_{e}\left(\mathbf{x}^{\prime}\right)\right) d \mathbf{s}^{\prime} \\
& +\frac{1}{\delta} \oint_{S} \frac{\gamma_{0}\left(\mathbf{x}^{\prime}\right)}{1+\alpha_{0}\left(\mathbf{x}^{\prime}\right)} G\left(\mathbf{x}, \mathbf{x}^{\prime}\right)\left[\frac{\partial \psi_{0}}{\partial \mathbf{x}^{\prime}} \times\left(\mathbf{b}_{0}\left(\mathbf{x}^{\prime}\right)+\mathbf{b}_{e}\left(\mathbf{x}^{\prime}\right)\right)\right] d \mathbf{s}^{\prime} \\
& +\frac{\partial}{\partial \tau} \oint_{S} G\left(\mathbf{x}, \mathbf{x}^{\prime}\right) \mathbf{a}\left(\mathbf{x}^{\prime}\right) d \mathbf{s}^{\prime} .
\end{aligned}
$$

Преобразуя поверхностные интегралы в интегралы по объему $D$ и учитывая формулу (27), получим окончательное выражение для потенциала $\psi$ :

$$
\begin{aligned}
& \psi(\mathbf{x})=\frac{1}{I_{T}} \sum_{m=1}^{M} I_{m} \oint_{S} G\left(\mathbf{x}, \mathbf{x}^{\prime}\right) g_{m}\left(\mathbf{x}^{\prime}\right) d s^{\prime} \\
& +\int_{D} \frac{\gamma_{0}\left(\mathbf{x}^{\prime}\right)\left(\nu \mathbf{B}_{e}\left(\mathbf{x}^{\prime}\right)-\mathbf{b}_{0}\left(\mathbf{x}^{\prime}\right)\right)}{1+\alpha_{0}\left(\mathbf{x}^{\prime}\right)}\left[\frac{\partial G\left(\mathbf{x}, \mathbf{x}^{\prime}\right)}{\partial \mathbf{x}^{\prime}} \frac{\partial \psi_{0}}{\partial \mathbf{x}^{\prime}}\right] d^{3} x^{\prime} \\
& +\int_{D} \frac{\partial G\left(\mathbf{x}, \mathbf{x}^{\prime}\right)}{\partial \mathbf{x}^{\prime}}\left\{\frac{\partial \mathbf{a}\left(\mathbf{x}^{\prime}\right)}{\partial \tau}-\frac{1}{1+\alpha_{0}\left(\mathbf{x}^{\prime}\right)}\left(\beta_{0}\left(\mathbf{x}^{\prime}\right) \nu \mathbf{B}_{e}\left(\mathbf{x}^{\prime}\right)\right.\right. \\
& \left.\left.-\beta_{0}\left(\mathbf{x}^{\prime}\right) \mathbf{b}_{0}\left(\mathbf{x}^{\prime}\right)+\alpha_{0}\left(\mathbf{x}^{\prime}\right) \frac{\partial \psi_{0}}{\partial \mathbf{x}^{\prime}}\right)\right\} d^{3} x^{\prime}+C .
\end{aligned}
$$

\section{Измеряемые потенциалы и взаимность}

Формула (30) совместно с формулами (27) и (28) позволяет получить потенциал внутри и на поверхности многополюсника. Однако места контактов, к которым прикреплены проводники, не могут рассматриваться как точечные. Измеряемое напряжение при этом равно разности потенциалов между сечениями проводников, подводимых к многополюснику, выбранными на некотором расстоянии от активной зоны элемента.
Пусть к контакту с номером k подключен измеритель напряжения, через который в общую шину течет ток $I_{k}$. Будем считать, что через боковую поверхность провода ток не течет, а вольтметр подключен к достаточно удаленному от активной зоны преобразователя сечению, которое является эквипотенциальным. Мощность тепловых потерь в подводящем проводе с учетом условия $\operatorname{div}(\mathbf{j})=0$ и формул $(21)$ и (22) имеет вид

$$
\begin{aligned}
\frac{d Q}{d t} & =l_{T}^{3} \int_{C k} \mathbf{j}(\mathbf{x}) \mathbf{E}(\mathbf{x}) d^{3} x \\
& =I_{k}\left(\varphi_{k}-\oint_{S} \varphi(\mathbf{x}) g_{k}(\mathbf{x}) d s\right)=I_{k}^{2} R_{U k} .
\end{aligned}
$$

Здесь $C_{k}$ - внутренняя область проводника, подсоединенного к $k$-й площадке, $R_{U k}-$ его омическое сопротивление, $\varphi_{k}$ - потенциал на эквипотенциальном сечении проводника, удаленном от магнитоактивной зоны. Отсюда

$$
\varphi_{k}-\oint_{S} \varphi(\mathbf{x}) g_{k}(\mathbf{x}) d s=I_{k} R_{U k} .
$$

Для потенциала, измеряемого идеальным вольтметром при токе $I_{k}$, стремящемся к нулю, получаем

$$
\varphi_{k}=\varphi_{T} \oint_{S} g_{k}(\mathbf{x}) \psi(\mathbf{x}) d s .
$$

Используя соотношения (31) и (22), получим

$$
\varphi_{k}\left(\mathbf{I}, \mathbf{B}_{e}\right)=\sum_{m=1}^{M} R_{k m}\left(\mathbf{I}, \mathbf{B}_{e}\right) I_{m}+\sum_{m=1}^{M} L_{k m} \frac{d I_{m}}{d t}+C
$$

где в приближении малого поля в соответствии с формулами (30)

$$
\frac{\beta_{0}\left(\mathbf{e}_{0}, \mathbf{b}_{0}\right)}{1+\alpha_{0}\left(\mathbf{e}_{0}, \mathbf{b}_{0}\right)}=h_{1} \mathbf{e}_{0} \mathbf{e}_{0}+h_{2} \mathbf{e}_{0} \mathbf{b}_{0}+h_{3} \mathbf{b}_{0} \mathbf{b}_{0}+\ldots
$$

Тогда

$$
\begin{gathered}
R_{k m}\left(\mathbf{I}, \mathbf{B}_{e}\right)=R_{k m}^{A}+R_{k m}^{H}\left(\mathbf{B}_{e}\right)+R_{k m}^{N L}\left(\mathbf{I}, \mathbf{B}_{e}\right), \\
R_{k m}^{A}=R_{T} \oint_{S} \oint_{S} g_{k}(\mathbf{x}) g_{m}\left(\mathbf{x}^{\prime}\right) G\left(\mathbf{x}, \mathbf{x}^{\prime}\right) d s d s^{\prime}, \\
R_{k m}^{H}\left(\mathbf{B}_{e}\right)=v R_{T} \int_{D} \mathbf{B}_{e}\left(\mathbf{x}^{\prime}\right) \oint_{S} \oint_{S}\left[\frac{\partial G\left(\mathbf{x}^{\prime}, \mathbf{x}^{\prime \prime}\right)}{\partial \mathbf{x}^{\prime}}\right. \\
\left.\times \frac{\partial G\left(\mathbf{x}, \mathbf{x}^{\prime}\right)}{\partial \mathbf{x}^{\prime}}\right] g_{k}(\mathbf{x}) g_{m}\left(\mathbf{x}^{\prime \prime}\right) d s d s^{\prime \prime} d^{3} x^{\prime}, \\
R_{k m}^{N L}(\mathbf{I})=\overbrace{R}^{N L}(\mathbf{I})+\breve{R}_{k m}^{N L}(\mathbf{I}),
\end{gathered}
$$




$$
\begin{aligned}
& \widehat{R}_{k m}^{N L}(\mathbf{I})=R_{T} \int_{D} \frac{\gamma_{0}\left(\mathbf{x}^{\prime}, \mathbf{I}\right) \mathbf{b}_{0}\left(\mathbf{x}^{\prime}, \mathbf{I}\right)}{1+\alpha_{0}\left(\mathbf{x}^{\prime}, \mathbf{I}\right)} \\
& \times \oint_{S} \oint_{S}\left[\frac{\partial G\left(\mathbf{x}^{\prime}, \mathbf{x}^{\prime \prime}\right)}{\partial \mathbf{x}^{\prime}} \frac{\partial G\left(\mathbf{x}, \mathbf{x}^{\prime}\right)}{\partial \mathbf{x}^{\prime}}\right] g_{k}(\mathbf{x}) g_{m}\left(\mathbf{x}^{\prime \prime}\right) d s d s^{\prime \prime} d^{3} x^{\prime} \\
& +R_{T} \int_{D} \frac{\alpha_{0}\left(\mathbf{x}^{\prime}, \mathbf{I}\right)}{1+\alpha_{0}\left(\mathbf{x}^{\prime}, \mathbf{I}\right)} \oint_{S} \oint_{S} \frac{\partial G\left(\mathbf{x}^{\prime}, \mathbf{x}^{\prime \prime}\right)}{\partial \mathbf{x}^{\prime}} \\
& \times \frac{\partial G\left(\mathbf{x}, \mathbf{x}^{\prime}\right)}{\partial \mathbf{x}^{\prime}} g_{k}(\mathbf{x}) g_{m}\left(\mathbf{x}^{\prime \prime}\right) d s d s^{\prime \prime} d^{3} x^{\prime}+\frac{\delta R_{T}}{4 \pi} \\
& \times \int_{D} \int_{D} \frac{\beta_{0}\left(\mathbf{x}^{\prime}, \mathbf{I}\right)}{1+\alpha_{0}\left(\mathbf{x}^{\prime}, \mathbf{I}\right)} \oint_{S} \oint_{S} \frac{\mathbf{x}^{\prime}-\mathbf{x}^{\prime \prime \prime}}{\left|\mathbf{x}^{\prime}-\mathbf{x}^{\prime \prime \prime}\right|^{3}}\left[\frac{\partial G\left(\mathbf{x}^{\prime}, \mathbf{x}^{\prime \prime}\right)}{\partial \mathbf{x}^{\prime}}\right. \\
& \left.\times \frac{\partial G\left(\mathbf{x}, \mathbf{x}^{\prime}\right)}{\partial \mathbf{x}^{\prime}}\right] g_{k}(\mathbf{x}) g_{m}\left(\mathbf{x}^{\prime \prime}\right) d s d s^{\prime \prime} d^{3} x^{\prime} d x^{\prime \prime \prime}, \\
& \breve{R}_{k m}^{N L}(\mathbf{I})=v R_{T} \int_{D}\left\{1-\frac{\gamma_{0}\left(\mathbf{x}^{\prime}, \mathbf{I}\right)}{1+\alpha_{0}\left(\mathbf{x}^{\prime}, \mathbf{I}\right)}\right\} \\
& \times \mathbf{B}_{e}\left(\mathbf{x}^{\prime}\right) \oint_{S} \oint_{S}\left[\frac{\partial G\left(\mathbf{x}^{\prime}, \mathbf{x}^{\prime \prime}\right)}{\partial \mathbf{x}^{\prime}} \frac{\partial G\left(\mathbf{x}, \mathbf{x}^{\prime}\right)}{\partial \mathbf{x}^{\prime}}\right] \\
& \times g_{k}(\mathbf{x}) g_{m}\left(\mathbf{x}^{\prime \prime}\right) d s d s^{\prime \prime} d^{3} x^{\prime}-v R_{T} \\
& \times \int_{D}\left\{h_{1} \mathbf{e}_{0}\left(\mathbf{x}^{\prime}\right)+h_{2} \mathbf{b}_{0}\left(\mathbf{x}^{\prime}\right)\right\} \mathbf{B}_{e}\left(\mathbf{x}^{\prime}\right) \\
& \times \oint_{S} \oint_{S} \frac{\partial G\left(\mathbf{x}^{\prime}, \mathbf{x}^{\prime \prime}\right)}{\partial \mathbf{x}^{\prime}} \frac{\partial G\left(\mathbf{x}, \mathbf{x}^{\prime}\right)}{\partial \mathbf{x}^{\prime}} \\
& \times g_{k}(\mathbf{x}) g_{m}\left(\mathbf{x}^{\prime \prime}\right) d s d s^{\prime \prime} d^{3} x^{\prime}-\frac{\nu R_{T} \delta}{4 \pi} \\
& \times \int_{D} \int_{D} h_{3} \mathbf{b}_{0}\left(\mathbf{x}^{\prime}\right) \mathbf{B}_{e}\left(\mathbf{x}^{\prime}\right) \oint_{S} \oint_{S} \frac{\mathbf{x}^{\prime}-\mathbf{x}^{\prime \prime \prime}}{\left|\mathbf{x}^{\prime}-\mathbf{x}^{\prime \prime \prime}\right|^{3}} \\
& \times\left[\frac{\partial G\left(\mathbf{x}^{\prime}, \mathbf{x}^{\prime \prime}\right)}{\partial \mathbf{x}^{\prime}} \frac{\partial G\left(\mathbf{x}, \mathbf{x}^{\prime}\right)}{\partial \mathbf{x}^{\prime}}\right] \\
& \times g_{k}(\mathbf{x}) g_{m}\left(\mathbf{x}^{\prime \prime}\right) d s d s^{\prime \prime} d^{3} x^{\prime} d^{3} x^{\prime \prime \prime}, \\
& L_{k m}=\frac{\mu_{0} l_{T}}{4 \pi} \int_{D} \int_{D} \oint_{S} \oint_{S} \frac{\partial G\left(\mathbf{x}^{\prime \prime \prime}, \mathbf{x}^{\prime \prime}\right)}{\partial x^{\prime \prime \prime}} \frac{\partial G\left(\mathbf{x}, \mathbf{x}^{\prime}\right)}{\partial x^{\prime}} \\
& \times \frac{g_{k}(\mathbf{x}) g_{m}\left(\mathbf{x}^{\prime \prime}\right)}{\left|\mathbf{x}^{\prime}-\mathbf{x}^{\prime \prime \prime}\right|} d s d s^{\prime \prime} d^{3} x^{\prime} d x^{\prime \prime \prime}
\end{aligned}
$$

и обозначено $R_{T}=\varphi_{T} / I_{T}$. Тогда из уравнений (34) и (29) непосредственно следует, что

$$
R_{k m}^{A}=R_{m k}^{A} .
$$

Преобразуя внутренний двойной поверхностный интеграл в уравнении (35) в произведение поверхностного интеграла по переменной $\mathbf{x}^{\prime \prime}$ на поверхностный интеграл по переменной $\mathbf{x}$, получим с учетом условия (28)

$$
R_{k m}^{H}=v R_{T} \int_{D} \mathbf{B}_{e}\left(\mathbf{x}^{\prime}\right)\left[\boldsymbol{\Gamma}_{m}\left(\mathbf{x}^{\prime}\right) \times \boldsymbol{\Gamma}_{k}\left(\mathbf{x}^{\prime}\right)\right] d^{3} x^{\prime},
$$

где обозначено

$$
\boldsymbol{\Gamma}_{m}\left(\mathbf{x}^{\prime}\right)=\oint_{S} \frac{\partial G\left(\mathbf{x}, \mathbf{x}^{\prime}\right)}{\partial \mathbf{x}^{\prime}} g_{m}(\mathbf{x}) d s
$$

Из соотношения (41) видно, что

$$
R_{k m}^{H}\left(\mathbf{B}_{e}(\mathbf{r})\right)=R_{m k}^{H}\left(-\mathbf{B}_{e}(\mathbf{r})\right)=-R_{m k}^{H}\left(\mathbf{B}_{e}(\mathbf{r})\right) .
$$

Аналогичными преобразованиями интегралы в правой части формул (37)-(39) с учетом условия (28) приводятся к виду

$$
\begin{aligned}
& \widehat{R}_{k m}^{N L}=R_{T} \int_{D} \frac{\gamma_{0}\left(\mathbf{x}^{\prime}, \mathbf{I}\right) \mathbf{b}_{0}\left(\mathbf{x}^{\prime}, \mathbf{I}\right)}{1+\alpha_{0}\left(\mathbf{x}^{\prime}, \mathbf{I}\right)}\left[\boldsymbol{\Gamma}_{m}\left(\mathbf{x}^{\prime}\right) \times \boldsymbol{\Gamma}_{k}\left(\mathbf{x}^{\prime}\right)\right] d^{3} x^{\prime} \\
& +R_{T} \int_{D} \frac{\alpha_{0}\left(\mathbf{x}^{\prime}, \mathbf{I}\right)}{1+\alpha_{0}\left(\mathbf{x}^{\prime}, \mathbf{I}\right)} \boldsymbol{\Gamma}_{m}\left(\mathbf{x}^{\prime}\right) \boldsymbol{\Gamma}_{k}\left(\mathbf{x}^{\prime}\right) d^{3} x^{\prime}+\frac{\delta R_{T}}{4 \pi} \\
& \times \int_{D} \int_{D} \frac{\beta_{0}\left(\mathbf{x}^{\prime}, \mathbf{I}\right)}{1+\alpha_{0}\left(\mathbf{x}^{\prime}, \mathbf{I}\right)} \frac{\mathbf{x}^{\prime}-\mathbf{x}^{\prime \prime}}{\left|\mathbf{x}^{\prime}-\mathbf{x}^{\prime \prime}\right|^{3}}\left[\boldsymbol{\Gamma}_{m}\left(\mathbf{x}^{\prime}\right) \times \boldsymbol{\Gamma}_{k}\left(\mathbf{x}^{\prime \prime \prime}\right)\right] d^{3} x^{\prime} d^{3} x^{\prime \prime}, \\
& \quad \breve{R}_{k m}^{N L}=v R_{T} \int_{D} \frac{1+\alpha_{0}\left(\mathbf{x}^{\prime}, \mathbf{I}\right)-\gamma_{0}\left(\mathbf{x}^{\prime}, \mathbf{I}\right)}{1+\alpha_{0}\left(\mathbf{x}^{\prime}, \mathbf{I}\right)} \mathbf{B}_{e}\left(\mathbf{x}^{\prime}\right) \\
& \quad \times\left[\int_{D}\left(\mathbf{x}^{\prime}\right) \times \boldsymbol{\Gamma}_{k}\left(\mathbf{x}^{\prime}\right)\right] d^{3} x^{\prime}+v R_{T} \\
& \quad-\frac{\left.v R_{T} \delta \mathbf{e}_{0}\left(\mathbf{x}^{\prime}\right)+h_{2} \mathbf{b}_{0}\left(\mathbf{x}^{\prime}\right)\right\} \mathbf{B}_{e}\left(\mathbf{x}^{\prime}\right) \boldsymbol{\Gamma}_{m}\left(\mathbf{x}^{\prime}\right) \boldsymbol{\Gamma}_{k}\left(\mathbf{x}^{\prime}\right) d^{3} x^{\prime}}{4 \pi} \int_{D} \mathbf{b}_{0}\left(\mathbf{x}^{\prime}\right) \mathbf{B}_{e}\left(\mathbf{x}^{\prime}\right) \frac{\mathbf{x}^{\prime}-\mathbf{x}^{\prime \prime}}{\left|\mathbf{x}^{\prime}-\mathbf{x}^{\prime \prime}\right|^{3}} \\
& \quad \times\left[\boldsymbol{\Gamma}_{m}\left(\mathbf{x}^{\prime}\right) \times \boldsymbol{\Gamma}_{k}\left(\mathbf{x}^{\prime \prime \prime}\right)\right] d^{3} x^{\prime} d^{3} x^{\prime \prime}, \\
& L_{k m}=L_{T} \int_{D} \int_{D} \frac{\boldsymbol{\Gamma}_{m}\left(\mathbf{x}^{\prime}\right) \boldsymbol{\Gamma}_{k}\left(\mathbf{x}^{\prime \prime \prime}\right)}{\left|\mathbf{x}^{\prime}-\mathbf{x}^{\prime \prime}\right|} d^{3} x^{\prime} d^{3} x^{\prime \prime}=L_{m k},
\end{aligned}
$$

где обозначено $L_{T}=\frac{\mu_{0} l_{T}}{4 \pi}$. Тогда с учетом условия (31) получаем

$$
R_{k m}^{N L}\left(\mathbf{I}, \mathbf{B}_{e}\right)=R_{m k}^{N L}\left(-\mathbf{I},-\mathbf{B}_{e}\right) .
$$

Из соотношений (40), (43) и (45) следует условие взаимности для матрицы нелинейных сопротивлений многополюсника в неоднородном внешнем магнитном поле

$$
R_{k m}\left(\mathbf{I}, \mathbf{B}_{e}(\mathbf{r})\right)=R_{m k}\left(-\mathbf{I},-\mathbf{B}_{e}(\mathbf{r})\right) .
$$




\section{Методика измерений}

Формула (33) представляет матрицу нелинейных сопротивлений многополюсника в виде суммы матрицы линейных сопротивлений (34), элементы которой не зависят ни от токов, ни от внешнего поля, матрицы линейных сопротивлений (35), элементы которой пропорциональны внешнему магнитному полю и не зависят от токов, и матрицы нелинейных сопротивлений (36), элементы которой зависят от токов, но не зависят от внешнего магнитного поля. Однако провести экспериментальную проверку непосредственно соотношений (40), (43) и (45) невозможно, так как потенциалы $\varphi_{k}$ в формуле (32) отсчитаны от среднего по поверхности тела потенциала, который сам зависит от втекающих в тело токов.

Выберем произвольный фиксированный путь $C_{k n}$, проходящий между $k$-м и $n$-м контактами многополюсника вне него. Тогда с учетом формул (19), (29) и (32) для напряжения $U_{k n}$ между этими зажимами получаем

$$
\begin{aligned}
& U_{k n}\left(\mathbf{I}, \mathbf{B}_{e}\right)=\int_{C_{k n}} \mathbf{E}(\mathbf{r}) d \mathbf{r}=\varphi_{k}-\varphi_{n}-\frac{d}{d t} \int_{C_{k n}} A(\mathbf{r}) d \mathbf{r} \\
& =\sum_{m=1}^{M}\left\{R_{k m}\left(\mathbf{I}, \mathbf{B}_{e}\right)-R_{n m}\left(\mathbf{I}, \mathbf{B}_{e}\right)\right\} I_{m} \\
& +\sum_{m=1}^{M}\left\{L_{k m}-L_{n m}-L_{k n m}\right\} \frac{d I_{m}}{d t}+U_{k n}^{e} .
\end{aligned}
$$

Здесь

$$
L_{k n m}=L_{T} \int_{C_{k n}} \int_{D} \frac{\boldsymbol{\Gamma}_{m}\left(\mathbf{x}^{\prime}\right)}{\left|\mathbf{x}-\mathbf{x}^{\prime}\right|} d \mathbf{x} d^{3} x^{\prime}, \quad U_{k n}^{e}=\frac{d}{d t} \int_{C_{k n}} \mathbf{A}_{e}(\mathbf{r}) d \mathbf{r}
$$

- не зависящее от токов $I_{m}$ напряжение, создаваемое внешним переменным магнитным полем $\mathbf{B}_{e}$. Слагаемые $U_{k n}^{e}$ в уравнениях (47) можно исключить, повторив измерения напряжений в холостом режиме при нулевом токе через контакты.

На практике постоянные токи и напряжения задаются и измеряются гораздо точнее, чем переменные. Поэтому экспериментальную проверку соотношений (40), (43) и (45) следует проводить в режиме постоянного тока, например для преобразователя Холла, геометрия которого приведена на рис. 1.

Проверку соотношений взаимности для нелинейного четырехполюсника целесообразно проводить тем же методом, как и для линейного - при разрыве на одной из сторон в двух режимах измерений:

1) Контакты 1 и 3 подключены к источнику тока $i_{k}$, между контактами 2 и 4 измеряется напряжение $u_{1}=\varphi_{2}^{(1)}-\varphi_{4}^{(1)}$, при этом $I_{1}=i_{k}, I_{3}=-i_{k}, I_{2}=I_{4}=0$.

2) Контакты 2 и 4 подключены к источнику тока $i_{k}$, между контактами 1 и 3 измеряется напряжение $u_{2}=\varphi_{1}^{(2)}-\varphi_{3}^{(2)}$, при этом $I_{2}=i_{k}, I_{4}=-i_{k}, I_{1}=I_{3}=0$.

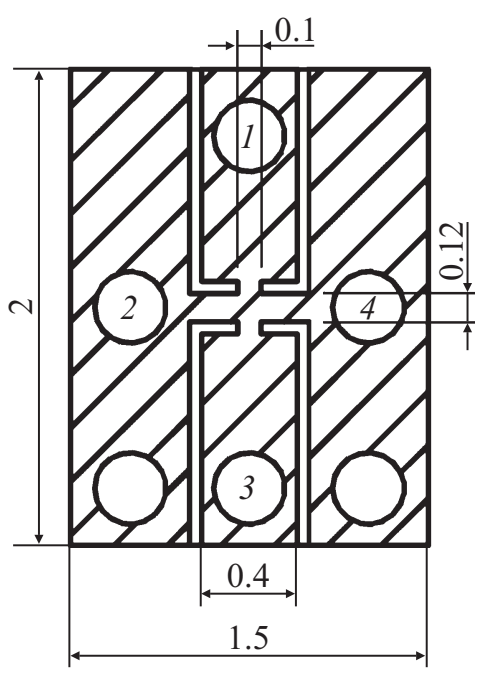

Рис. 1. Геометрия преобразователя Холла; размеры приведены в миллиметрах.

Измерения в режимах 1 и 2 выполняются при нескольких значения тока $i_{k}, k=1, \ldots, K,-i_{0} \leq I_{k} \leq i_{0}$, по их результатам методом наименьших квадратов аппроксимируются полиномами ВАХ

$$
\begin{gathered}
\tilde{u}_{1}(i)=\left\{u_{1}(i)-u_{1}(-i)\right\} / 2=a_{1} i+a_{2} i^{3}+\ldots, \\
\tilde{u}_{2}(i)=\left\{u_{2}(i)-u_{2}(-i)\right\} / 2=b_{1} i+b_{2} i^{3}+\ldots .
\end{gathered}
$$

и строятся функции

$$
\begin{gathered}
\tilde{R}(i)=\frac{\tilde{u}_{1}(i)}{i}=a_{1}+a_{2} i^{2}+a_{3} i^{4}+\ldots, \\
\tilde{R}_{2}(i)=\frac{\tilde{u}_{2}(i)}{i}=b_{1}+b_{2} i^{2}+b_{3} i^{4}+\ldots .
\end{gathered}
$$

При этом число отсчетов $K$ должно в несколько раз превышать степень полиномов аппроксимации.

Для слабого внешнего поля $\left|\mathbf{B}_{e}\right| \ll 1 / v$ справедливо линейное приближение

$$
R_{k m}^{N L}\left(\mathbf{I}, \mathbf{B}_{e}\right)=R_{m k}^{N L}(\mathbf{I})+\mathbf{H}_{k m}(\mathbf{I}) \mathbf{B}_{e} .
$$

Тогда из формулы (46) следует, что

$$
R_{m k}^{N L}(\mathbf{I})=R_{m k}^{N L}(-\mathbf{I}), \quad \mathbf{H}_{k m}(\mathbf{I})=-\mathbf{H}_{m k}(\mathbf{I}) .
$$

Из формул (32) и (33), в свою очередь, следует, что

$$
\begin{aligned}
a_{1}\left(\mathbf{B}_{e}\right)= & R_{21}^{(1)}\left(\mathbf{B}_{e}\right)-R_{23}^{(1)}\left(\mathbf{B}_{e}\right)+R_{43}^{(1)}\left(\mathbf{B}_{e}\right) \\
& -R_{41}^{(1)}\left(\mathbf{B}_{e}\right)=R_{1}^{A}+R_{1}^{H}\left(\mathbf{B}_{e}\right), \\
b_{1}\left(\mathbf{B}_{e}\right)= & R_{12}^{(1)}\left(\mathbf{B}_{e}\right)-R_{14}^{(1)}\left(\mathbf{B}_{e}\right)+R_{34}^{(1)}\left(\mathbf{B}_{e}\right) \\
& -R_{32}^{(1)}\left(\mathbf{B}_{e}\right)=R_{2}^{A}+R_{2}^{H}\left(\mathbf{B}_{e}\right), \\
R_{1}^{A}= & R_{21}^{A}-R_{23}^{A}+R_{43}^{A}-R_{41}^{A}, \\
R_{2}^{A}= & R_{12}^{A}-R_{14}^{A}+R_{34}^{A}-R_{32}^{A},
\end{aligned}
$$




$$
\begin{aligned}
& R_{1}^{H}\left(\mathbf{B}_{e}\right)=R_{21}^{H}\left(\mathbf{B}_{e}\right)-R_{23}^{H}\left(\mathbf{B}_{e}\right)+R_{43}^{H}\left(\mathbf{B}_{e}\right)-R_{41}^{H}\left(\mathbf{B}_{e}\right), \\
& R_{2}^{H}\left(\mathbf{B}_{e}\right)=R_{12}^{H}\left(\mathbf{B}_{e}\right)-R_{14}^{H}\left(\mathbf{B}_{e}\right)+R_{34}^{H}\left(\mathbf{B}_{e}\right)-R_{32}^{H}\left(\mathbf{B}_{e}\right) .
\end{aligned}
$$

Из формул (40), (43) и (45) следует, что

$$
R_{1}^{A}=R_{2}^{A}=R_{A}, \quad R_{1}^{H}\left(\mathbf{B}_{e}\right)=-R_{2}^{H}\left(\mathbf{B}_{e}\right)=R_{H}\left(\mathbf{B}_{e}\right),
$$

где

$$
R_{A}=\frac{a_{1}\left(\mathbf{B}_{e}\right)+b_{1}\left(\mathbf{B}_{e}\right)}{2}, \quad R_{H}\left(\mathbf{B}_{e}\right)=\frac{a_{1}\left(\mathbf{B}_{e}\right)-b_{1}\left(\mathbf{B}_{e}\right)}{2} .
$$

Соотношения (52) нельзя проверить при одном значении тока, так как по результатам измерения двух величин $u_{1}$, $u_{2}$ не возможно определить четыре величины $R_{1}^{A}, R_{2}^{A}, R_{1}^{H}$, $R_{2}^{H}$. Но, согласно условию (50), функции

$$
\begin{gathered}
r_{1}(i)=\tilde{R}_{1}(i)-a_{1}=a_{2} i^{2}+a_{3} i^{4}+\ldots, \\
r_{2}(i)=\tilde{R}_{2}(i)-b_{1}=b_{2} i^{2}+b_{3} i^{4}+\ldots
\end{gathered}
$$

от внешнего магнитного поля $\mathbf{B}_{e}$ не зависят. Поэтому при выполнении соотношений (40), (43) и (45) из формул (51) и (54) следует, что величина $R_{A}$ не зависит ни от тока $i$, ни от внешнего магнитного поля $\mathbf{B}_{e}$, а величина $R_{H}$ не зависит от тока $i$ и пропорциональна внешнему магнитному полю $\mathbf{B}_{e}$. Поэтому оценкой точности выполнения соотношений (52) может служить относительное среднеквадратичное отклонение величин $R_{A}$ и $R_{H}$, полученное по $K$ значениям тока

$$
\begin{gathered}
\bar{R}_{A / H}=\frac{1}{K} \sum_{k=1}^{K} R_{A / H}\left(i_{k}\right) . \\
\delta\left[R_{A / H}\right]=\sqrt{\frac{1}{K} \sum_{k=1}^{K}\left(R_{A / H}\left(i_{k}\right)-\bar{R}_{A / H}\right)^{2}} .
\end{gathered}
$$

\section{Эксперимент}

Экспериментальная проверка соотношений взаимности производилась для преобразователей Холла серии ПХЭ фирмы ООО „Сенсор“, имеющих высокую крутизну преобразования порядка $1 \mathrm{~V} / \mathrm{T}$ и существенную нелинейность ВАХ. Для измерения ВАХ в экспериментальной установке использовались прецизионный измеритель напряжения и однополярный источник стабильного тока. Для обеспечения измерений при отрицательных значениях тока через преобразователь Холла использовалась система коммутации направления тока. Для проверки влияния магнитного поля на коэффициенты $a_{1}\left(\mathbf{B}_{e}\right)$ и $b_{1}\left(\mathbf{B}_{e}\right)$, определенных в формуле $(51)$, использовались кольца Гельмгольца с источником стабильного тока. Для уменьшения влияния геомагнитного поля на результаты измерений преобразователь Холла и кольца Гельмгольца были помещены в центр пермаллоевого экрана толщиной $1.5 \mathrm{~mm}$, выполненный в виде отрезка трубы диаметром $140 \mathrm{~mm}$ и длинной $405 \mathrm{~mm}$. На торцах

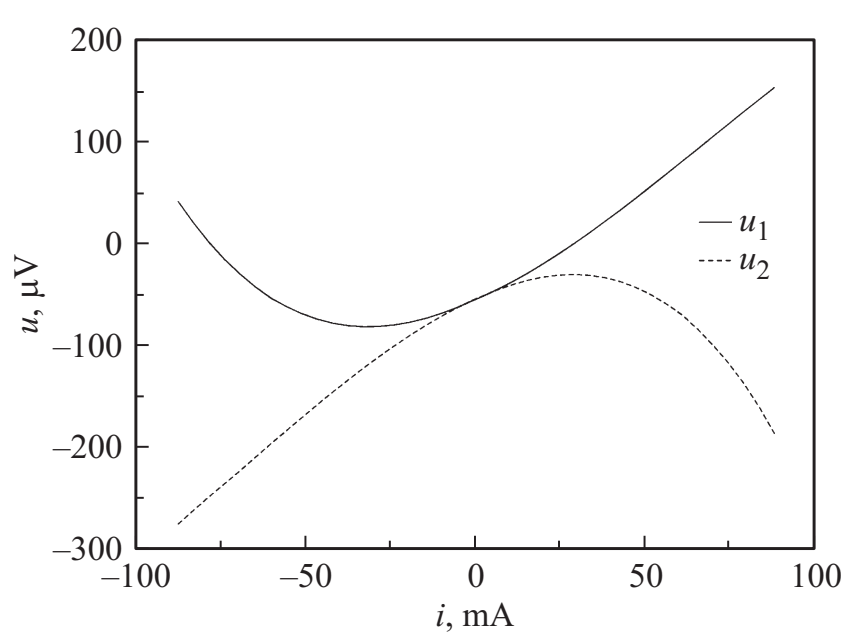

Рис. 2. Экспериментальные зависимости напряжений на потенциальных и токовых контактах от протекающего через датчик тока.

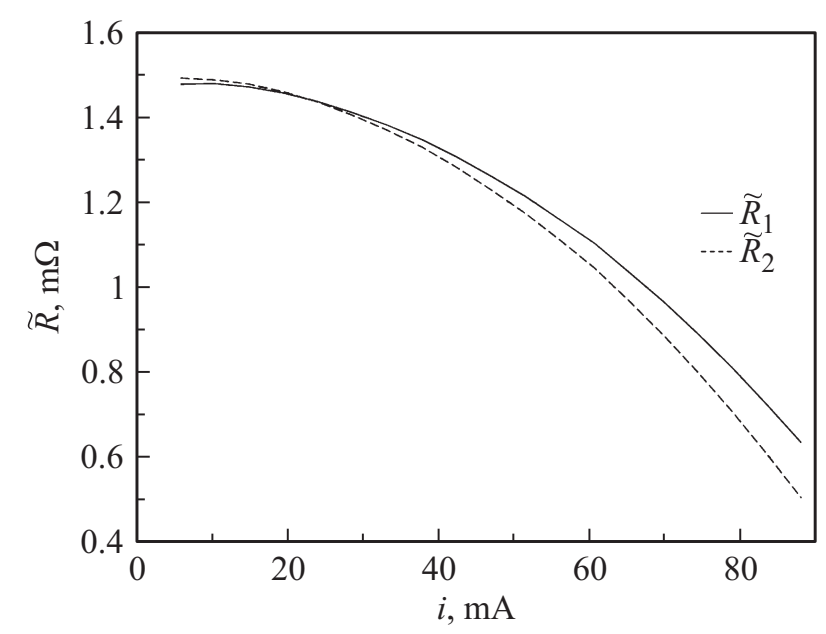

Рис. 3. Экспериментальные зависимости функций $\tilde{R}_{1}$ и $\tilde{R}_{2}$ от протекающего через датчик тока.

трубы были установлены заглушки, выполненные из магнитомягкой стали толщиной $8 \mathrm{~mm}$. Экспериментальная установка позволяет измерять напряжения с потенциальных и токовых контактов преобразователя в диапазоне $-500-500 \mu \mathrm{V}$ и среднеквадратичном отклонении порядка $10 \mathrm{nV}$, при токе через преобразователь $1-100 \mathrm{~mA}$ и индукции внешнего магнитного поля до $1 \mathrm{mT}$.

Для проверки соотношений взаимности использовался преобразователь Холла ПХЭ 606117А. Сначала были измерены зависимости напряжений $u_{1}(i)$ и $u_{2}(i)$ от протекающего через преобразователь Холла тока при отключенных кольцах Гельмгольца. Из приведенных на рис. 2 результатов видно, что исследуемый преобразователь обладает значительной нелинейностью ВАХ в допустимом диапазоне управляющих токов. Напряжение смещения установки порядка $-50 \mu \mathrm{V}$ не влияет на вычисляемые по формуле (48) величины. Далее по 


\begin{tabular}{r|c|c|c|c|c|c|c|c}
\hline $\begin{array}{c}B_{e}, \\
\mu \mathrm{T}\end{array}$ & $\begin{array}{c}\tilde{R}_{1}(0), \\
\mathrm{m} \Omega\end{array}$ & $\begin{array}{c}\tilde{R}_{1}\left(i_{0}\right), \\
\mathrm{m} \Omega\end{array}$ & $\begin{array}{c}\tilde{R}_{2}(0), \\
\mathrm{m} \Omega\end{array}$ & $\begin{array}{c}R_{2}\left(i_{0}\right), \\
\mathrm{m} \Omega\end{array}$ & $\begin{array}{c}R_{A}, \\
\mathrm{~m} \Omega\end{array}$ & $\begin{array}{c}\delta\left[R_{A}\right], \\
\mathrm{m} \Omega\end{array}$ & $\begin{array}{c}R_{H}, \\
\mathrm{~m} \Omega\end{array}$ & $\begin{array}{c}\delta\left[R_{H}\right], \\
\mathrm{m} \Omega\end{array}$ \\
\hline 0 & 1.437 & 0.634 & 1.436 & 0.504 & 1.521 & 0.001 & -0.010 & 0.000 \\
-10 & 1.315 & 0.502 & 1.508 & 0.581 & 1.493 & 0.004 & -0.106 \\
+10 & 1.511 & 0.709 & 1.316 & 0.386 & 1.495 & 0.003 & 0.087 & 0.000 \\
-50 & 1.035 & 0.245 & 1.785 & 0.860 & 1.496 & 0.004 & -0.384 & 0.001 \\
+50 & 1.791 & 0.988 & 1.037 & 0.112 & 1.498 & 0.003 & 0.365 \\
-100 & 0.688 & -0.109 & 2.135 & 1.197 & 1.493 & 0.002 & -0.732 & 0.001 \\
+100 & 2.134 & 1.333 & 0.685 & -0.234 & 1.495 & 0.003 & 0.713 & 0.002 \\
-300 & -0.701 & -1.478 & 3.539 & 2.597 & 1.503 & 0.003 & -2.125 & 0.006 \\
+300 & 3.532 & 2.714 & -0.706 & -1.617 & 1.497 & 0.002 & 2.103 & 0.006
\end{tabular}

формуле (49) были построены зависимости функции $\tilde{R}_{1}(i)$ и $\tilde{R}_{2}(i)$ от протекающего через преобразователь Холла тока, представленные на рис. 3.

Полученные зависимости были аппроксимированы полиномом пятого порядка согласно выражению (49), и по полученным данным построены корректировочные зависимости (54). С использованием этих зависимостей по формуле (53) были найдены сопротивления Холла $R_{H}$ и асимметрии $R_{A}$. В диапазоне рабочих токов 25-100 mА полученные сопротивления не зависят от тока с относительной погрешностью порядка $7 \cdot 10^{-4}$, что соответствует погрешности производимых измерений.

Далее были проведены измерения при наличии внешнего магнитного поля $\mathbf{B}_{e}$, создаваемого кольцами Гельмгольца. При анализе данных использовались полиномы, полученные на этапе калибровки. Значения функций (49) при нулевом и максимальном токах, а также рассчитанные по формулам (53) и (54) значения сопротивлений Холла $R_{H}$ и асимметрии $R_{A}$ и их среднеквадратичные отклонения от среднего приведены в таблице.

При аппроксимации методом наименьших квадратов зависимости сопротивления Холла от индукции внешнего магнитного поля получена крутизна преобразования $7.1 \Omega /$ T. Сопротивление Холла при нулевом токе в кольцах Гельмгольца, равное $0.010 \mathrm{~m} \Omega$, позволяет оценить остаточное геомагнитное поле внутри экрана как $1.5 \mu \mathrm{T}$. Сопротивление асимметрии при изменении направления поля не изменяется с точностью до погрешности измерений $R_{A}\left(\mathbf{B}_{e}\right) \sim R_{A}\left(-\mathbf{B}_{e}\right)$.

\section{Обсуждение результатов}

Соотношение (14) является материальным уравнением для изотропной среды и применимо к системам, к которым применимо кинетическое уравнение (1), предполагающее отсутствие турбулентностей и постоянные концентрации всех сортов носителей заряда [8]. Поэтому приведенный вывод неприменим в режиме лавинного пробоя и межзонных переходов. Кроме того, вывод соотношений взаимности неприменим к неустойчивому режиму, когда решение уравнения (10) может быть не единственным. Поэтому соотношение (16) и вытекаю- щие из него соотношения взаимности (46) могут не выполняться в сверхсильных полях из-за осцилляций, турбулентности и других нестабильностей [8].

Метод последовательных приближений, использованный для решения уравнений (18), справедлив при $\delta=\mu_{0} \sigma v \varphi T<1$. Характерным при комнатной температуре значениям $\sigma=200(\Omega \cdot \mathrm{m})^{-1}, v=50 \mathrm{~m}^{2} /(\mathrm{V} \cdot \mathrm{s})$ соответствует значение $\delta=3.3 \cdot 10^{-3}$.

Значительная нелинейность измеренных вольт-амперных характеристик преобразователя (рис. 2), при которой $u(-i) \neq-u(i)$, обусловлена в основном магнитным самовоздействием токов. При управляющем токе $100 \mathrm{~mA}$ создаваемое им внутреннее магнитное поле в пленке преобразователя (рис. 1) может достигать $\sim 0.5 \mathrm{mT}$, что в 300 раз больше остаточного магнитного поля внутри экрана. Уменьшение сопротивления $\tilde{R}(i)$ в 3 раза при увеличении управляющего тока до $100 \mathrm{~mA}$ связано с увеличением проводимости арсенида индия при нагреве проводящей пленки преобразователя током. Рассеиваемая преобразователем мощность при управляющем токе $100 \mathrm{~mA}$ достигает $150 \mathrm{~mW}$. Различие зависимостей $\tilde{R}_{1}(i)$ и $\tilde{R}_{2}(i)$ (рис. 3 ) обусловлено не симметричной геометрией преобразователя (рис. 1). Изменение сопротивления асимметрии для различных значений магнитного поля (таблица), по-видимому, связано с изменением температуры окружающей среды на $1^{\circ} \mathrm{C}$ при проведении эксперимента. Согласно паспортным данным преобразователя Холла ПХЭ606117А, температурный дрейф его сопротивления асимметрии может достигать $0.050 \mathrm{~m} \Omega /{ }^{\circ} \mathrm{C}$, что хорошо согласуется с полученными результатами.

\section{Заключение}

Формулы (46) показывают, что классические соотношения взаимности [2] выполнятся в квазистационарном случае для нелинейного многополюсника, находящегося во внешнем неоднородном магнитном поле даже при наличии существенной нелинейности. Они могут применяться для оптимизации режимов работы элементов функциональной электроники [10] и их сигнальных характеристик. В частности, системная функция преоб- 
разователя вида (42) не зависит от режима работы преобразователя, определяется только его геометрией и может быть найдена численно. В этом случае ее можно рассматривать как дополнительную паспортную характеристику преобразователя и использовать для компенсации возникающих искажений сигнала путем редукции [11]. Из формул (51) следует, что способ уменьшения погрешностей холловского магнитометра, основанный на использовании линейных соотношений взаимности в скрещенных полях [2], будет справедлив и для высокочувствительного преобразователя Холла с нелинейной ВАХ. Его применение позволит повысить точность магнитных измерений и выделить линейный отклик многополюсника на слабое внешнее неоднородное магнитное поле $\mathbf{B}_{e}$ на фоне значительно более сильного магнтиного поля $\mathbf{B}^{\prime}$, создаваемого протекающими по элементу токами.

Работа выполнена за счет гранта РНФ № 15-1900028 „Разработка методики магнитного структурного анализа и гибридной экспертной системы оперативной технической диагностики металлических изделий в геомагнитном поле“.

\section{Список литературы}

[1] Игнатьев А.А., Ляшенко А.В. Магнитоэлектроника СВЧ-, КВЧ-диапазонов в пленках ферритов. М.: Наука, 2005. $380 \mathrm{c}$.

[2] Голубев А.А., Игнатьев В.К., Никитин А.В. // ПТЭ. 2008. T. 51. № 5. C. 53-58.

[3] Lars Onsager // Phys. Rew. 1931. Vol. 37. P. 405-426.

[4] Игнатьев В.К., Орлов А.А., Перченко С.В. // Письма в ЖТФ. 2016. Т. 42. № 4. C. 74-81.

[5] IgnatjeV., Orlov A., Perchenko S. // Progress In Electromagnetics Res. Lett. 2016. Vol. 59. P. 71-75.

[6] Дьярмати И. Неравновесная термодинамика. Теория поля и вариационные принципы. М.: Мир, 1974. 304 с.

[7] Де Гроот С.P. Термодинамика необратимых процессов. М.: ГИТТЛ, 1956. $281 \mathrm{c.}$

[8] Электродинамика плазмы / Под ред. А.И. Ахиезера. М.: Наука, 1974. $720 \mathrm{c}$.

[9] Свешников А.Г., Боголюбов А.Н., Кравцов В.В. Лекции по математической физике. М.: Изд-во МГУ, 1993. 352 с.

[10] Игнатьев А.А., Ляшенко А.В. Гетеромагнитная микроэлектроника. Микросистемы активного типа. М.: Наука, 2006. $877 \mathrm{c}$.

[11] Игнатьев В.К., Протопопов А.Г. // Известия вузов. Приборостроение. 2003. Т. 46. № 3. С. 38-44. 